\title{
Harnessing the Electron Beam to Study Reactions in Graphene Liquid Cells and Degradation in Sensitive 2D Materials
}

\author{
Sarah Haigh, David Hopkinson, Daniel Kelly, Nick Clark, Yichao Zou and Roman Gorbachev \\ University of Manchester, Manchester, England, United Kingdom
}

The number of 2D materials continues to expand rapidly although many of those with the most the promising magnetic or optoelectronic properties being found to be unstable in air. These materials can be stabilised in ambient conditions by exfoliating the crystal in an inert argon glove box and encapsulating it with stable 2D layers, such as graphene and hexagonal boron nitride. [1] This encapsulation approach also provides an ideal platform for high resolution scanning transmission electron microscopy (STEM) investigations, with the high conductivity of encapsulating graphene sheets having been shown to reduce the rate of electron beam damage for the encapsulating crystal for both STEM and TEM imaging [ref Kaiser paper]. Using an accelerating voltage below the threshold for knock-on damage in the encapsulating graphene, $<80 \mathrm{kV}$, preserves the encapsulation over repeated imaging experiments. Alternatively, imaging with higher accelerating voltages allows focused damage to the encapsulation layer by the STEM electron probe. In black phosphorus this can be harnessed to enable highly localised oxidation of the encapsulated material, facilitating high spatial resolution patterning of device architectures [3]. We find that the electron beam conditions optimised in the STEM can be reproduced in electron beam lithography to enable patterning of encapsulated black phosphorous crystals with a sub $\sim 10$ $\mathrm{nm}$ resolution, better than is achievable using conventional polymeric resists.[3]

We have applied the encapsulation approach to study the presence and behaviour of point and extended defects in the 2D monochalcogenides, GaSe and InSe. These materials have shown relatively high electron mobilities, strong second harmonic generation in monolayers and a direct bandgap in bulk form which transitions to a quasi-direct bandgap when reduced to the monolayer limit.[4] These properties have been successfully applied in few-layer photodetection and field effect devices and when used together in $\mathrm{GaSe} / \mathrm{InSe}$ heterostructures have shown tunable photoemission from interlayer excitons. The performance of devices based on GaSe and InSe can be affected by the presence of impurities or by exposure to air, light, and/or moisture, yet, little has been done to characterise the structure of these defects. We have used atomic resolution STEM together with electron energy loss spectroscopy (EELS) and energy dispersive X-ray spectroscopy to characterise individual point and extended defects in these materials [4]. By analysing annular dark field (ADF) STEM images of mono-, bi-, and few-layer GaSe and InSe crystals we observe that these materials can form both metal and selenium vacancies under the action of the electron beam. Selenium vacancies are observed to be healable: recovering the perfect lattice structure in the presence of selenium or enabling incorporation of dopant atoms in the presence of impurities. At an electron beam energy of $80 \mathrm{keV}$, the elastic Mott scattering cross section is very small for all native atom species in these crystals and the maximum energy transferred by the beam is insufficient to exceed the displacement threshold energy for any of the elements both in the pristine crystal and at defect sites. It can thus be concluded that elastic damage mechanisms, such as knock-on or sputtering, are unlikely to dominate under these conditions and so we hypothesize that radiolysis and chemical reaction with impurity species trapped inside the encapsulation barrier is likely to be contributing to damage. Under prolonged imaging, multiple point defects are observed to coalesce to form extended defect structures, with GaSe generally developing trigonal defects and InSe primarily forming line defects; suggesting that both $\mathrm{Ga}$ and Se are chemically attacked in GaSe while Se is primarily susceptible in InSe. Extended vacancy defects and stacking faults are extensively found despite exfoliation the crystals in an argon glove 
box. This suggests that to maintain the perfect lattice structure of such materials may require exfoliation to be performed in ultra-high vacuum (UHV) conditions, and this UHV exfoliation technology has recently been experimentally realised. Furthermore, the stacking faults we observe have symmetries not found in bulk samples and which could be used to tune the properties of $2 \mathrm{D}$ post-transition-metal monochalcogenide materials for optoelectronic applications.

In addition, we will present work where 2D material heterostructure 'sandwiches' are enabling new in situ STEM imaging capabilities, where materials are imaged in liquid or gas environments. Here we show they can be used as a platform to initiate and study a wide variety of chemical reactions in liquid environments with unprecedented spatial resolution and unrivaled spectroscopic capabilities [5].
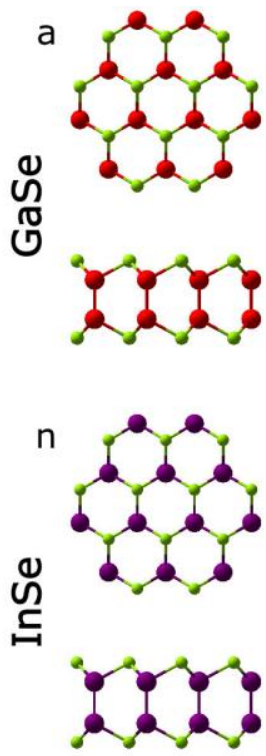
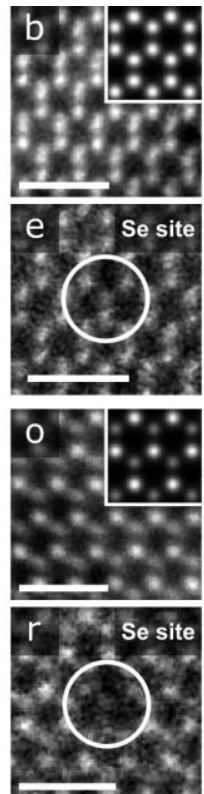
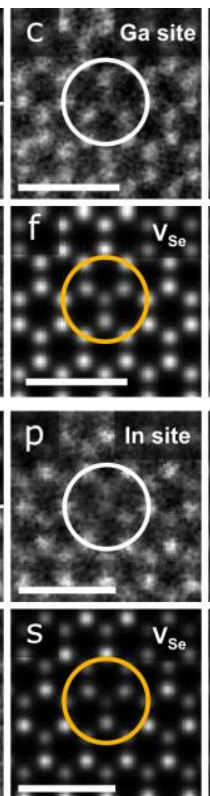
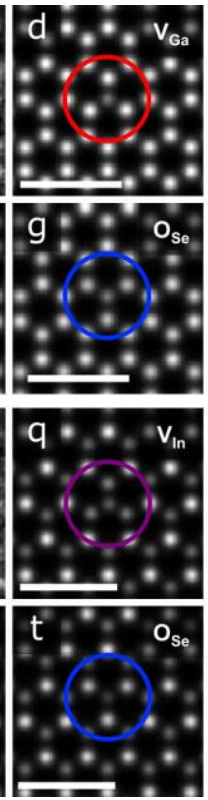
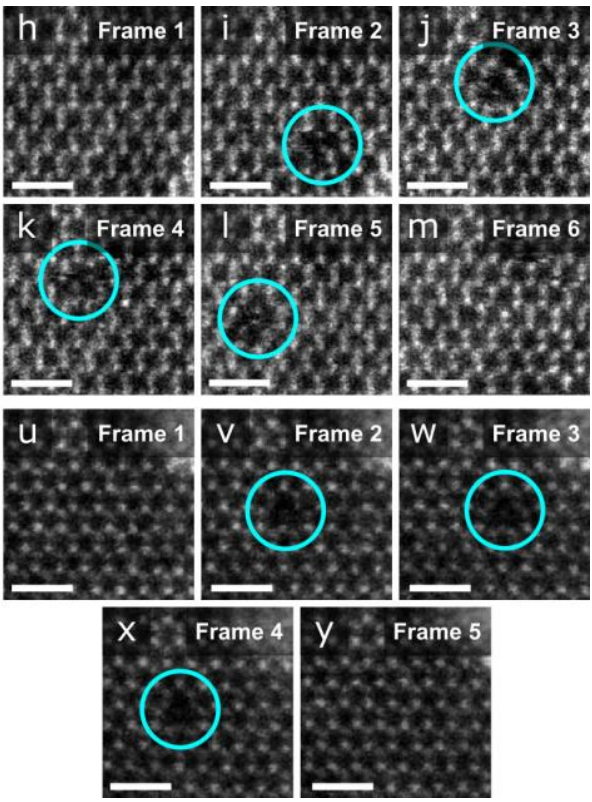

Figure 1. ADF-STEM images identifying point defects and their motion in monolayer GaSe (a-m) and InSe (n-y) crystals. Far left are shown schematics of the atomic structures. Left panels show high resolution images of the pristine structure with simulations inset (b) and (o). Images of point defects $(\mathrm{c}, \mathrm{e}, \mathrm{p}, \mathrm{r})$ are then compared to simulations (d,f,g,q,s,t) to identify the nature of the defects. In GaSe, Se vacancies are found to be substituted by an impurity (e.g. O) while in InSe, Se vacancies are not substituted. Right hand panels show frames from dynamic movies illustrating the movement and apparent healing of defects under the electron beam. All scale bars: $1 \mathrm{~nm}$. Adapted with permission from the American Chemical Society, reference [4], copyright 2019. 


\section{GaSe}
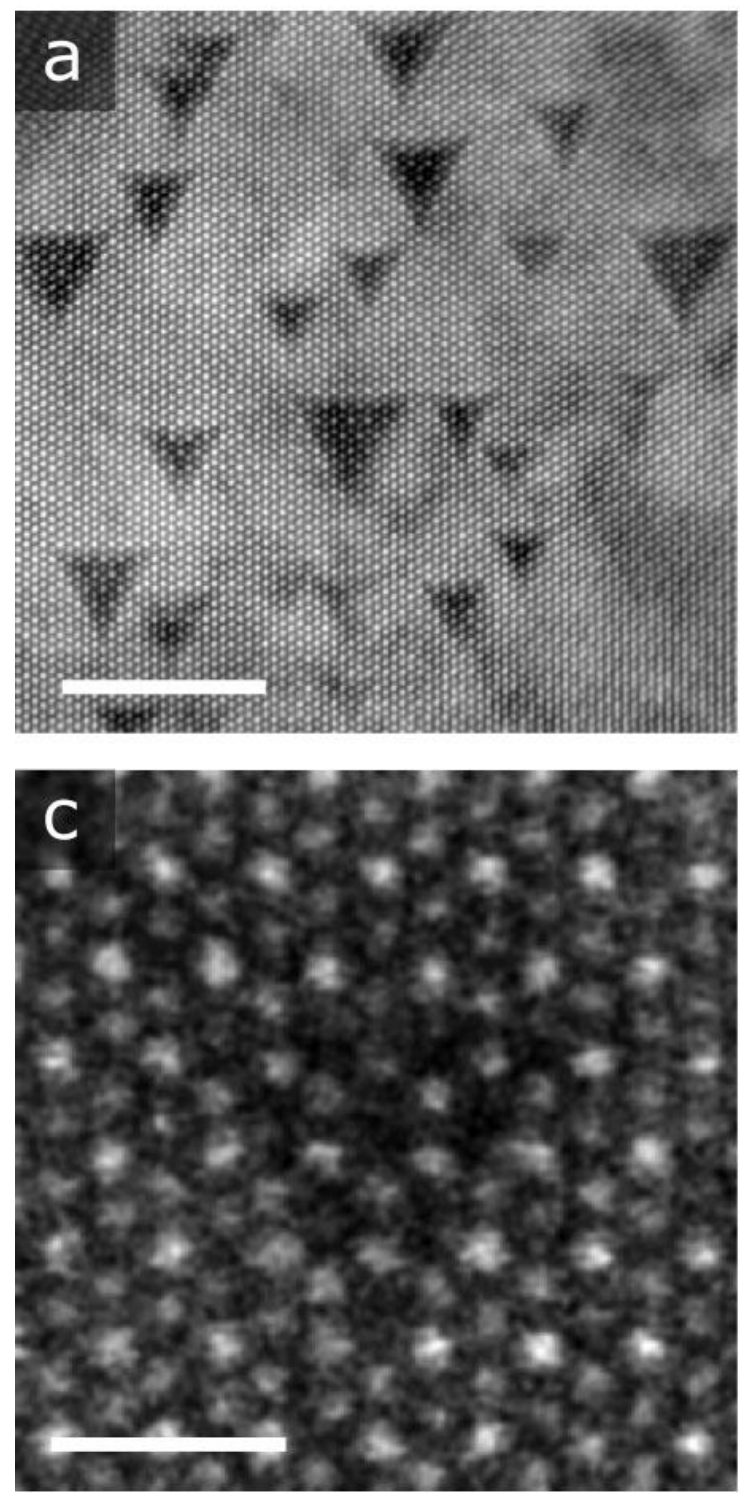

InSe
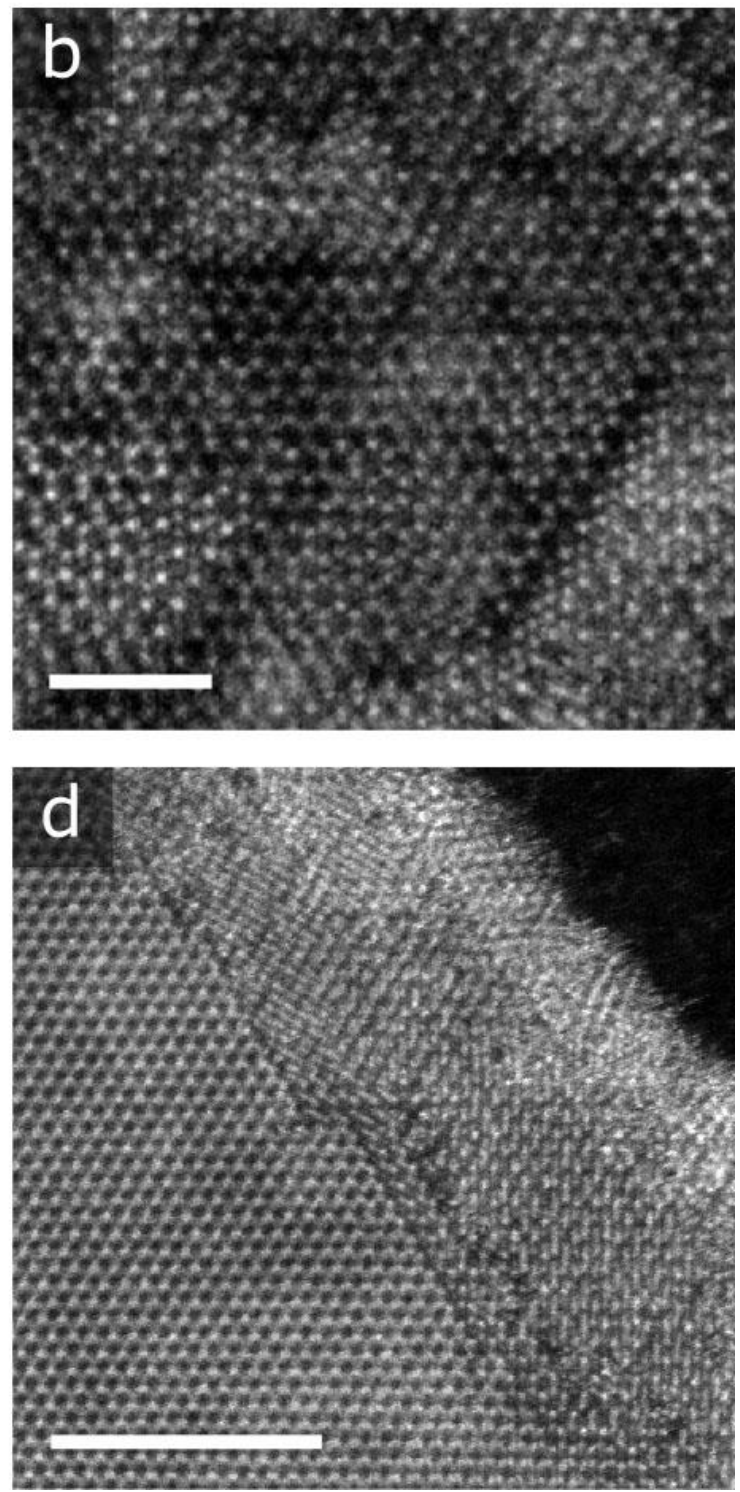

Figure 2. Comparison of extended defects observed in GaSe and InSe. (a) Time averaged image of stable trigonal defects in thick (>10 layer) GaSe. Scale bar: $5 \mathrm{~nm}$. (c) Detail of a trigonal defect in bilayer GaSe. Scale bar: $1 \mathrm{~nm}$. (b) Dense networks of line defects in bilayer InSe. Scale bar: $2 \mathrm{~nm}$. (d) Atomically sharp crystalline-amorphous transition towards the edge of a few layer InSe. Scale bar: $5 \mathrm{~nm}$. Adapted with permission from the American Chemical Society, ref [4], copyright 2019.

References

[1] Cao et al Quality Heterostructures from Two-Dimensional Crystals Unstable in Air by Their Assembly in Inert Atmosphere, Nano Lett. 2015, 15, 8, 4914-4921

[2] G Gerardo Algara-Siller et al. The pristine atomic structure of MoS2 monolayer protected from electron radiation damage by graphene, Appl. Phys. Lett. 103, 203107 (2013); https://doi.org/10.1063/1.4830036

[3] N. Clark et al Scalable Patterning of Encapsulated Black Phosphorus, Nano Letters, 2018, 18, 9, 53735381 
[4] D G Hopkinson et al, Formation and Healing of Defects in Atomically Thin GaSe and InSe, ACS Nano, (2019) 10.1021/acsnano.8b08253

[5] D J Kelly et al Nanometer Resolution Elemental Mapping in Graphene-Based TEM Liquid Cells, Nano Letters, (2018) 18, 2, 1168 